\title{
USINg ONTOlogy BASEd SEMANTIC ASSOCIATION Rule Mining IN Location BASEd SERVICES
}

\author{
Ali Mousavi ${ }^{1}$, Andrew Hunter ${ }^{1}$ and Mohammad Akbari ${ }^{2}$ \\ ${ }^{1}$ Department of Geomatics, University of Calgary, Calgary, Canada \\ ${ }^{2}$ Department of Civil Engineering, University of Birjand, Birjand, Iran
}

\begin{abstract}
Recently, GPS and mobile devices allowed collecting a huge amount of mobility data. Researchers from different communities have developed models and techniques for mobility analysis. But they mainly focused on the geometric properties of trajectories and do not consider the semantic facet of moving objects. The techniques are good at extracting patterns, but they are hard to interpret in a specific application domain. This paper proposes a methodology to understand mobility data and semantically interpret trajectory patterns. The process considers four different behavior types such as semantic, semantic and space, semantic and time, and semantic and space-time. Finally, a system prototype was developed to evaluate the behavior models in different aspects using one of the location based services. The results showed that applying the semantic association rules could significantly reduce the number of available services and customize the services based on the rules.
\end{abstract}

\section{KEYWORDS}

Data mining, ontology, semantic, location based services, association rule mining, spatiotemporal data

\section{INTRODUCTION}

With the increasing number of smartphones, it becomes handy to collect the movement data generated by different moving entities. Moreover, most of the smart phones can offer positioning functionalities such as Global Positioning System (GPS) [1]. These kinds of technologies significantly increase the potentials of existing applications such as traffic monitoring and establishing geo-social networks, and also promote accessing location based services (LBS) such as location-aware advertising and integrated information services [2]. The widespread use of these applications and services in our daily activities has led to a large number of positioning data that can be represented as trajectories [3]. Generally, these types of data take the form of an [x,y, $t$ ] triplet that represents the spatial coordinates and time stamp of a location [4]. With such an increase of trajectory data, there is a need for improving the existing methods to efficiently handle and investigate user behavior from such a large amount of data.

To explore user behavior, some researchers have investigated new analytical techniques [5]-[7] and computational methods [8]-[10] for the analysis of movement data. Moreover, some researchers have developed new techniques to discover similar trajectories [11], periodic movement [12], and identify relative motion patterns [13]. Therefore, many studies have determined approaches to use trajectory data for different types of knowledge discovery methods such as trajectory data modelling and analysis, trajectory pattern mining, and exploratory visual analytics [8], [9], [14], [15]. However, the proposed approaches have mainly concentrated on the geometric properties of trajectories. Therefore, the extracted patterns are classified based on a set of geometric properties. The techniques are good at extracting patterns, but the they are difficult to understand in a specific application domain [16]. In addition, when the nature of the movement

DOI : $10.5121 /$ ijdkp.2016.6501 
data is considered as context within the mining process, the mined results can be made more meaningful [3]. Therefore, movement data needs to be reconsidered from the spatiotemporal and semantic view as well. Recently, new research efforts have investigated methods that support trajectories with rich conceptual models where semantics of movement can be clearly expressed [17]-[20]. Considering former conceptual studies on trajectories, the motivation of this paper is to further explore semantic methods for analyzing trajectory data, not only from a geometric view, but also from a semantic perspective as well. This can be obtained by means of a semantic process, where raw trajectories are enhanced with semantic information and integrated with geographic knowledge encoded in an ontology. Using semantic within the knowledge discovery process in order to extract behavior patterns can help in the understanding of the movement of an object [3].

Therefore, the main idea of this paper is to propose a methodology to understand mobility data and semantically interpret trajectory patterns. To achieve this goal, it is necessary to define a semantic conceptual data model and a technique to use a rich model and extract meaningful trajectories from the movement data. The reminder of the paper is organized as follows. In section 2 related work from current work is presented. In section 3 the proposed methodology is described and each part is illustrated. In section 4 the conducted experiments on a dataset is showed. Moreover, a summary of the results is presented. In section 5 the paper is concluded and the future research regarding further development is described.

\section{RELATED WORKS}

\subsection{Knowledge Discovery for Movement Data}

Recently, the database community has concentrated on defining several spatiotemporal data types such as moving points and moving regions [21]. Guting et al [22] have paid significant attention to moving object databases. They proposed various methods for modeling moving objects in constraints networks such as road networks. Pelekis et al [23] established a prototype named HERMES to use the spatial data types, which were provided by a database management system. Mostly, research in this area has motivated on the geometric facet of trajectories. A number of data mining (DM) techniques have been proposed for discovering similar trajectories [24]-[26]. Generally, most research have concentrated on the mining step itself, and overlooked the entire knowledge discovery process [4].

Lately, the perception of a semantic trajectory was advanced as a sequence of stops and moves [27]. A stop is defined as an attracting place in which a moving object has stayed for a period of time and move is defined as the part of the object's movement among consecutive stops [21]. A number of researchers have implemented this concept as a standard for semantic trajectory modelling and analysis, but only a few such as Vania et al [4], Baglioni et al [28] and Trasarti et al [29] have adopted semantic knowledge to interpret and understand trajectory patterns. Therefore, in this work we extend a typical DM framework by considering ontologies in the knowledge discovery process.

\subsection{Ontology and semantic knowledge discovery}

Ontology is a key in geospatial information systems by defining interrelations between different domains of spatial and temporal entities [30]. Lately, some research have shown the efficiency of using ontologies in the knowledge discovery process [31]. Phillips and Buchanan [32] utilized ontologies to improve a feature selection method in the process of knowledge discovery. Bernstein et al. [33] proposed an ontology based intelligent DM to rank the mining process. 
Bauer and Baldes [34] designed an ontology based interface to help users understand their learning system from a semantic view. Canataro and Camito [35] expressed the use of an ontology based DM to simplify knowledge discovery process. However, none of the mentioned works have deliberated a model ontology that contains spatial, temporal, and other dimensions of data. They mostly concentrated on data dictionaries, data interoperability, and data representation [21]. Therefore, the main purpose of this paper is to demonstrate the key role that ontologies can play in the knowledge discovery process, especially in the association rule mining process.

\section{MeTHOdOLOGY}

Figure 1 illustrates the proposed methodology. It consists of three main parts, namely: semantic trajectory ontology, semantic behavior modelling, and prototype development.

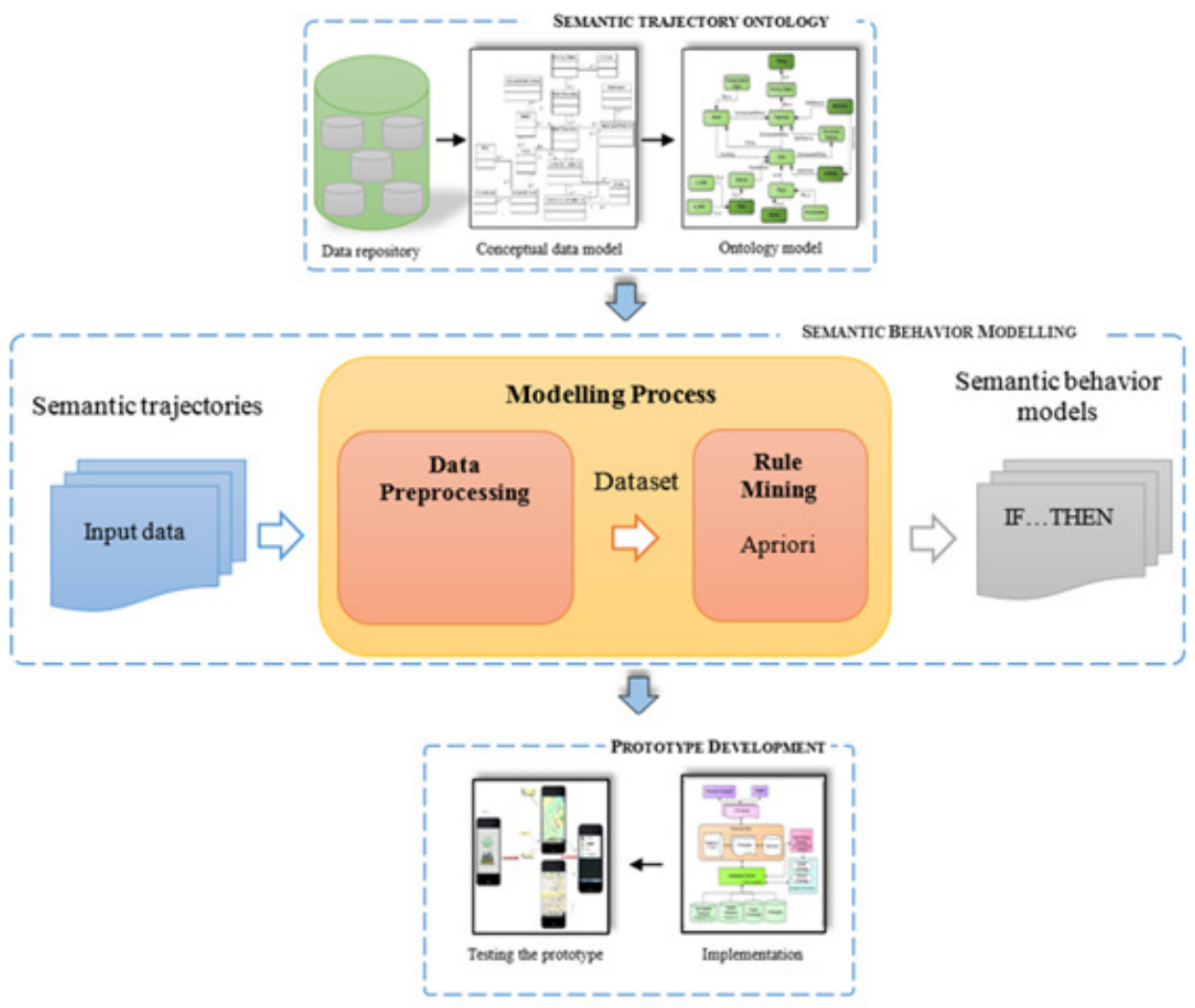

Figure 1. The proposed methodology

The first part generally contains maps/layers and an application domain. In this part, a conceptual data model is defined and based on that, a semantic trajectory ontology model is designed. Then the ontology concepts are mapped to the users' trajectory data. The second part shows the procedure to model the behavior of users. It includes data preprocessing and rule mining. The input data for the modelling process are semantic trajectories. The input data has to be preprocessed and transformed into a proper dataset that is suitable as an input for rule mining. The generated rules constitute the behavior model, which are imported into the ontology model. In the last part, a prototype is developed to evaluate the ontology based behavior model in different aspects by considering one of the LBSs called location based advertisement. 


\subsection{Semantic Trajectory Ontology}

The essential part of this work is a conceptual data model of semantic trajectories, which is illustrated in Figure 2-a. It describes the conceptual model, which addresses the modelling requirements with the goal of analysis of semantic trajectory data. The aim of this model is to represent the concepts and relations of the movement domain where trajectory data and semantic movement patterns are to be interpreted along with the behavior types. This model is an expanded form of the conceptual data model presented in Spaccapietra et al.[17]. The conceptual model contains information related to: moving object, raw trajectory, sub-trajectory, semantic subtrajectory, semantic trajectory, semantic place, stop, move, activity type, and behavior type.

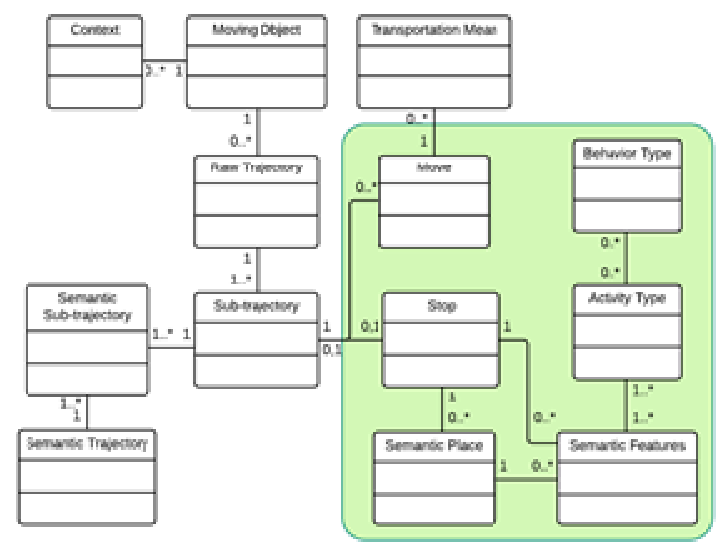

Figure 2. (a) Extended conceptual model of semantic trajectory used in this work

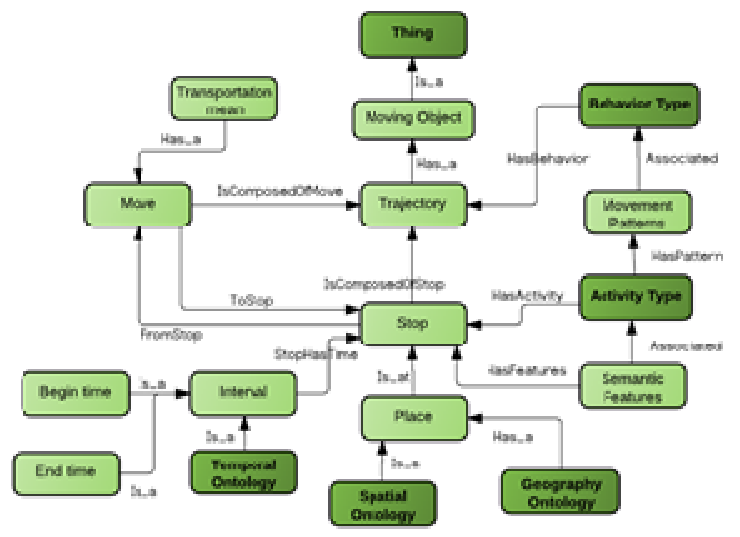

Figure 2. (b) Semantic trajectory ontology model

Moreover, in this part an ontology model is built based on the proposed conceptual data model. Figure 2-b shows a very partial version of this ontology with only the most important concepts and relationships. This ontology provides the semantic description of application-relevant trajectories with their domain specific semantic meaning. Every trajectory is composed of stops and every stop is connected to other stops by moves. Moves can be identified by transportation mean type. Every stop is connected to an interval that represents the time of the stop. It includes begin time and end time concepts that identify when the trajectory starts and ends. Moreover, 
each stop is linked to a place and an activity type that can be performed in the place in order to enrich the stop entity. An activity can be associated to a behavior type, which is already associated to the trajectory concept. Movement patterns are composed of different activity types, which are some of the main parameters to identify movement behavior.

\subsection{Ontology Based Semantic Behavior Modelling}

\subsubsection{Semantic trajectories}

A semantic trajectory is a type of trajectory that has been enhanced using contextual data such as activity types. The process of creating the semantic trajectories is described in detail in Mousavi and Hunter [36]. As shown in Table 1, a semantic trajectory of a user, which not only has the activity types but also has some other semantic features such as time, duration, and date. For instance, On June $8^{\text {th }}$, the user has left home at 7:12 am and has gone to work at 7:45 am and stayed there for 9 hours and 13 minutes.

Table 1. Semantic trajectory of a user

\begin{tabular}{ccccccc}
\hline Date & Start & Time_1 & End & Time_2 & Day & Stayed \\
\hline 8-Jun & Home & $7: 12$ & Work & $7: 45$ & Monday & $9: 13$ \\
\hline 8-Jun & Work & $17: 25$ & Shopping & $17: 41$ & Monday & $2: 01$ \\
\hline 8-Jun & Shopping & $19: 50$ & Home & $20: 13$ & Monday & $10: 12$ \\
\hline
\end{tabular}

The next step is to find the behavior type of users, which indicate the regularities between users' activity types. For instance, if a user goes shopping, what is the possibility the user might do other activity types such as going for shopping again, going to visit a friend or return home afterward (Figure 3). The figure shows a schematic representations of the activity types and their connections.

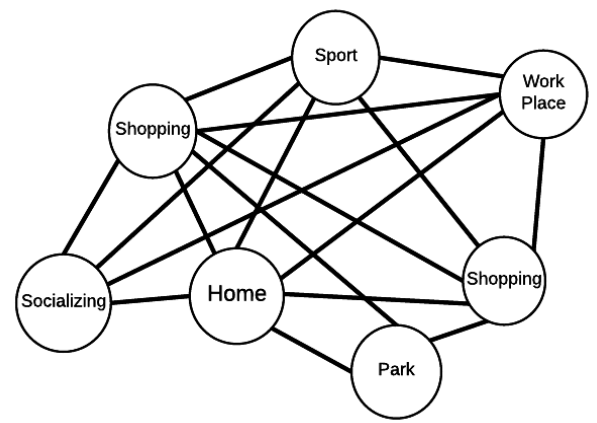

Figure 3. Association between different activity types

\subsubsection{Data preprocessing}

Data preprocessing is necessary to obtain an appropriate dataset. This process involves several steps such as selection, transformation and aggregation of the data. In this paper, ontologies are used for representing terms and relationships of the activities. Activities can be represented as taxonomy, from the more specific to the more general. Figure 4 shows an activity classification of a user. It is classified into four different major activities such as recreation, profession, shopping, and other activity types. 


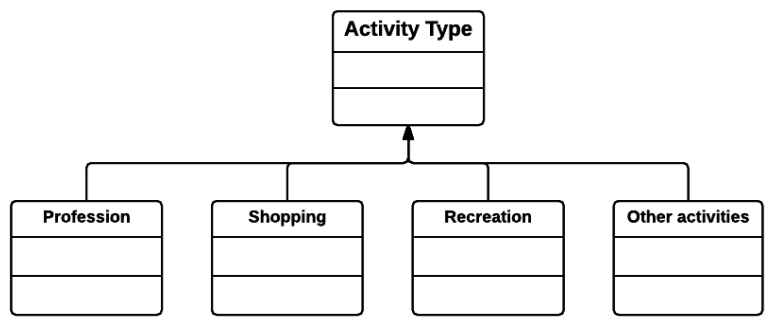

Figure 4. Activity types classification

Profession activity types could be working, getting involved into different jobs, etc., Shopping refers to time spent at different stores for buying food and drinks, or groceries in general required for one's daily needs. Recreation might include going to the theatre, the pub, gyms, and other places related to leisure. Other activity type might include relaxing at home, cultural or religious activities, etc. Humans regularly perform their activities at different times. Thus, it is very improbable to find regular relationships in a specific timestamp. This paper defines different ranges for date and time to extract some common behavior patterns. Therefore, the time ontology contains the temporal discretization such as absolute intervals as shown in Table 2.

Table 2. Temporal discretization of time ontology

\begin{tabular}{lc}
\hline & Time Definition \\
\hline Morning & 4:00 AM - 11:59 AM \\
\hline Afternoon & $12: 00$ PM - 4:59 PM \\
\hline Evening & 5:00 PM - 8:59 PM \\
\hline Night & $9: 00$ PM - 3:59 AM \\
\hline
\end{tabular}

The acquired data is used as input for the described mining process. Several datasets are generated based on variety of attributes. The behavior of a trajectory in general, is computed with intelligent methods such as data mining algorithms. As shown in Figure 5, a behavior is composed of different activities and their attributes, since one activity may or may not be followed by another.

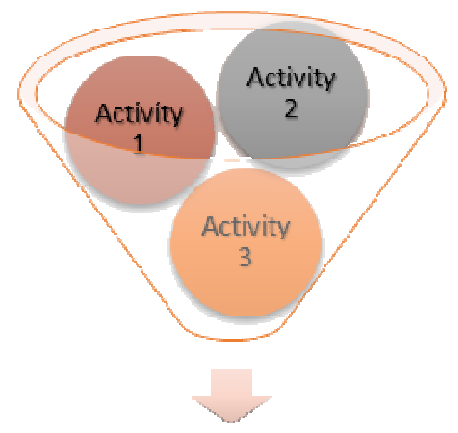

Behavior 1

Figure 5. A behavior is composed of different activities and their attributes 
Different types of behavior patterns such as semantic, semantic and time, semantic and space, and finally, semantic and space-time are considered as it can be seen in Figure 6.

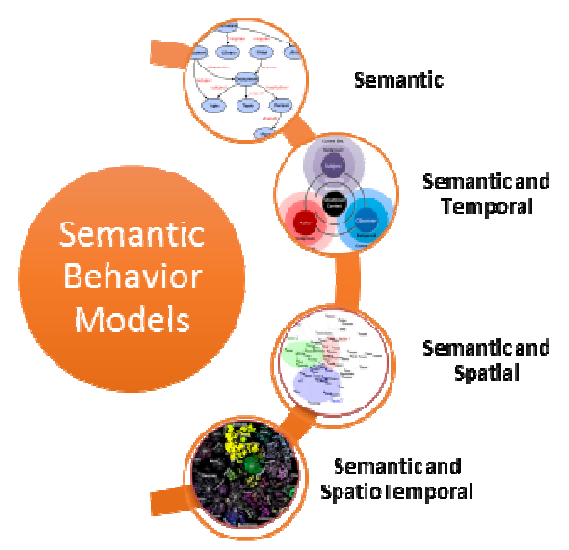

Figure 6. Different semantic behavior models

\subsubsection{Association Rule mining}

Apriori [37], which is one of the association rule mining algorithm was used to extract relationships from the observed activity types of users as behavior type. The dataset $D$ includes all different types of activities occurred by users and is used as input for the process. Let $A=\left\{a_{1}, \ldots, a_{n}\right\}$ be a finite set of all $n$ task relevant items. It is determined during the preprocessing, which was explained in the previous sub-section. A subset $X \subseteq A$ is named itemset. This algorithm is based on the frequency of the itemsets in the dataset. For instance, assume an instance $\mathrm{I}$ is a non-empty subset of $A: I \subseteq A$. All instances together form the dataset $D=\left\{I_{1}, \ldots, I_{n}\right\}$. An association rule is an implication of the form $X \Rightarrow Y$ where $X=A, Y=A, X \neq \varnothing, Y \neq \varnothing$ and $X \cap Y \neq \varnothing$. There are two measures to calculate the interestingness of the extracted patterns, which are called support and confidence [38].

The support (1) shows how often $X$ and $Y$ happened together in the dataset.

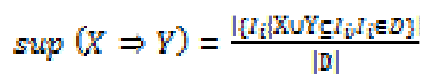

The confidence of a rule (2) shows the number of itemsets containing $\mathrm{Y}$ that also contains $\mathrm{X}$.

$$
\operatorname{conf}(X \Rightarrow Y)=\frac{\sup (X u Y)}{\operatorname{aup}(M)}
$$

The procedure is performed in two different steps. First, a set of frequencies is generated for each activity type. Minimum support criteria is applied as a constraint to control this step. Second, the relevant association rules are generated from the frequent itemsets. Minimum confidence criteria is applied to control and filter the expected rules from the itemsets. The minimum support and confidence criteria differ corresponding to the kinds of rules that the expert is willing to identify in the dataset[36]. 


\subsection{Prototype implementation}

In this work a prototype was developed to evaluate the applicability and usefulness of generated information using the proposed methodology. To test the prototype, location based advertisement, which is one of the LBSs, was considered. It is customized based on the results of the methodology. Therefore, several runs of the user based on different scenarios are tested.

\section{EXPERIMENTS}

The performance of the proposed methodology was evaluated using Calgary's dataset. The data used for this experiment was captured by a user, who has installed an application (developed for this research) on his phone and carried the phone with him while driving a car in the city for a year.

\subsection{Association rule mining}

Apriori algorithm was applied to determine support for, and confidence in a particular pattern to find rulesets. A number of association rules found in the Calgary's semantic trajectories using minimum support of 2 , and a minimum confidence of $60 \%$. All the extracted rulesets were stored into the data repository and imported into the ontology model. Different rulesets are shown below from different behavior types. Table 3 lists a number of rules extracted from semantic attributes. For instance, if the user performed a recreational activity, there was $2.3 \%$ support and $66.7 \%$ confidence that the user will return home or if the user performed a work activity, there was $26 \%$ support and $78 \%$ confidence that the user will return home.

Table 3. A number of extracted association rules developed from semantic attributes

\begin{tabular}{ccc}
\hline Rules & Support & Confidence \\
\hline If Recreational THEN Return home & $2.3 \%$ & $66.7 \%$ \\
\hline If Shopping THEN Return home & $22.1 \%$ & $62.1 \%$ \\
\hline If Work THEN Return home & $26 \%$ & $78 \%$ \\
\hline
\end{tabular}

Table 4 lists a number of rules extracted from semantic and time attributes. For instance, if the user performed a work activity in the afternoon, there was $3.1 \%$ support and $88.1 \%$ confidence that the user will return home or if the user was at home in the evening, there was $12.2 \%$ support and $75 \%$ confidence that the user will go shopping.

Table 4. A number of extracted association rules developed from semantic and time attributes

\begin{tabular}{ccc}
\hline Rules & Support & Confidence \\
\hline If Work - Afternoon THEN Return home & $3.1 \%$ & $88.1 \%$ \\
\hline If Home - Morning THEN Work & $26 \%$ & $94.1 \%$ \\
\hline If Home - Evening THEN Shopping & $12.2 \%$ & $75 \%$ \\
\hline
\end{tabular}

Table 5 lists a number of rules extracted from semantic and space attributes. For instance, if the user performed a shopping activity at location number 39, there was $6.1 \%$ support and $100 \%$ confidence that the user will return home or if the user was performed a socializing activity at location number 3 , there was $2.3 \%$ support and $100 \%$ confidence that the user will return home. 
International Journal of Data Mining \& Knowledge Management Process (IJDKP) Vol.6, No.5, September 2016

Table 5. A number of extracted association rules developed from semantic and space attributes

\begin{tabular}{ccc}
\hline Rules & Support & Confidence \\
\hline If Shopping_39 THEN Return home & $6.1 \%$ & $100 \%$ \\
\hline If Shopping_10 THEN Shopping_39 & $6.9 \%$ & $66.7 \%$ \\
\hline If Socializing_3 THEN Return home & $2.3 \%$ & $100 \%$ \\
\hline
\end{tabular}

Table 6 lists a number of rules extracted from semantic and space-time attributes. For instance, if the user performed a shopping activity at location number 39 in the evening, there was $5.3 \%$ support and $88.9 \%$ confidence that the user will return home or if the user was at home in the evening, there was $11.45 \%$ support and $68.5 \%$ confidence that the user will go shopping at location number 10 .

Table 6. A number of extracted association rules developed from semantic and space- time attributes

\begin{tabular}{ccc}
\hline Rules & Support & Confidence \\
\hline If Shopping_39 - Evening THEN Return home & $5.3 \%$ & $88.9 \%$ \\
\hline If Home - Evening THEN Shopping_10 & $11.45 \%$ & $68.5 \%$ \\
\hline If Socializing_3 - Evening THEN Return home & $2.3 \%$ & $100 \%$ \\
\hline
\end{tabular}

\subsection{Prototype Testing}

To test the capabilities of the system, several runs of the user based on different scenarios have been tested. Different cases are elaborated on below. Based on the user's semantic and time behavior ruleset, in the evening, the user usually performs a business activity (fuels with gas). Therefore, once the user passes by any gas station in the evening, he would get a notification to fuel with gas (Figure 7).

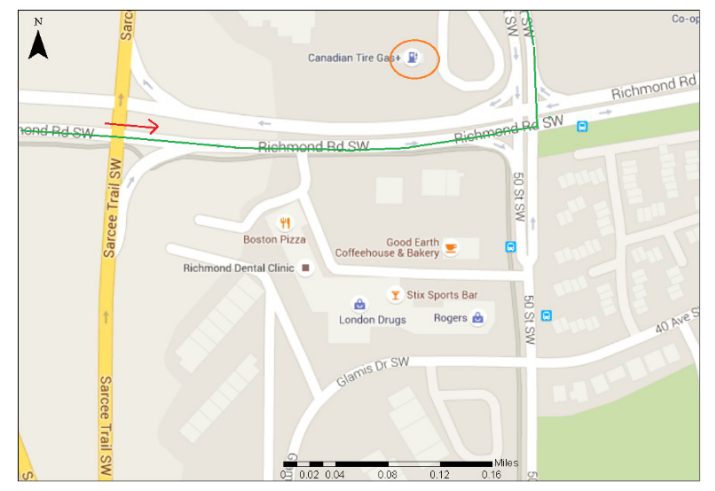

Figure 7. Testing the system using time and semantic behavior type

Based on the user's semantic behavior ruleset, since the user had some activities such as recreational, once he passes by a park, he would get a notification about the park (Figure 8). 
International Journal of Data Mining \& Knowledge Management Process (IJDKP) Vol.6, No.5, September 2016

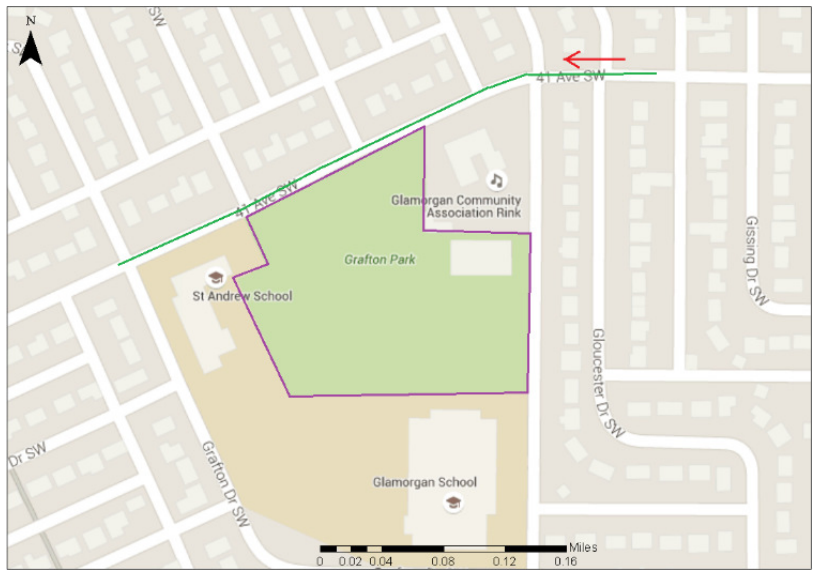

Figure 8. Testing the system using semantic behavior type

In this case, the user passed by two different places such as a park and a mall. Based on his semantic and space behavior ruleset, he would get two notifications, one for each location (Figure 9).

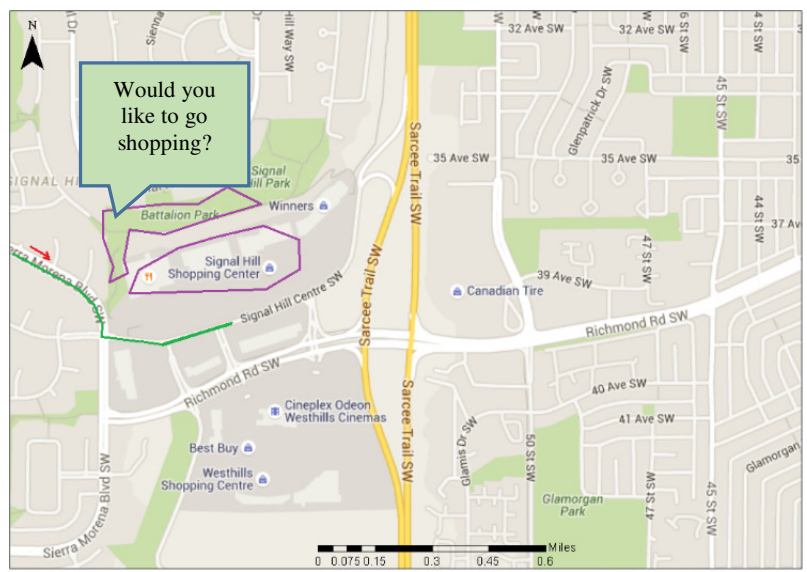

Figure 9. Testing the system using space and semantic behavior type

Based on the user's semantic and space-time behavior ruleset, if the user performs a recreational activity at location number 5 in the evening, then he would go shopping afterward. Therefore, based on this ruleset, the user has received a notification about a shopping activity while leaving the park and passing by a mall (Figure 10). 


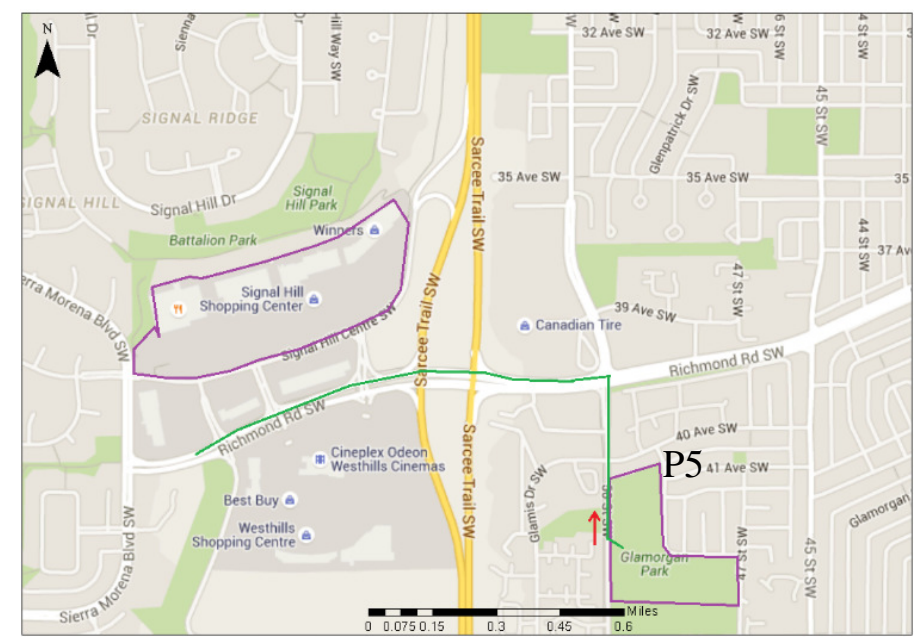

Figure 10. Testing the system using semantic and space-time behavior type

Moreover, the location based advertisement was used to test the extracted behavior models. Different service types; temporal, spatial and spatiotemporal were considered in this regard ( Figure 11. ). The temporal services are the ones with a limited time period. For instance, there's $30 \%$ discount on everything at Tommy this week. The spatial services are the ones that will be sent in specific distance of the user. For instance, if the user's distance to Tommy store is less than or equal to 200 meters, a notification will be sent to the user. The spatiotemporal services are the ones that not even the user has to be in specific distance of the store, but also it has to match with the time period. For instance, if the user's distance to Tommy store is less than or equal to 200 meters in this week, a notification will be sent to the user.

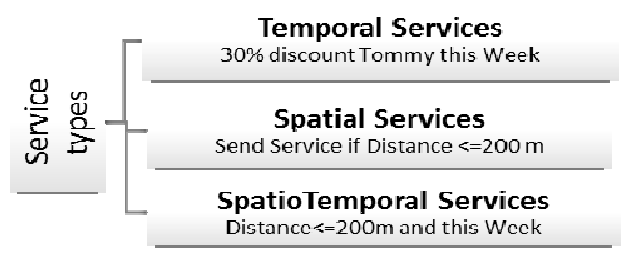

Figure 11. Service types

As it can be seen in Table 7, there are different service layers used in this research to implement the prototype such as sport, amenity, community center and business. Each one had variety of services to provide. For instance, amenity layer had some services related to parks and recreation.

Table 7. Different service categories of the city of Calgary

\begin{tabular}{cccc}
\hline Sport & Amenity & Community center & Business \\
\hline Education & Parks & Attraction & Retail dealer \\
School & Recreation & Community center & Contractor \\
& & Court & Cleaning services \\
& & Hospital & Food services \\
& & Library & Personal services \\
& & School & \\
\hline
\end{tabular}


As it can be seen in Table 8, the above mentioned services are related to the extracted activities. For instance, for recreational activities, two different service categories named sport and amenity were considered.

Table 8. Different service categories related to different activity types

\begin{tabular}{cc}
\hline Activity type & Service category \\
\hline Socializing & Sport/Amenity Services \\
\hline Shopping & Business Services \\
\hline Daily shopping & Business Services \\
\hline Eating & Business Services \\
\hline Education & Sport/Community Services \\
\hline Recreational & Sport/Amenity Services \\
\hline Business services & Business Services \\
\hline Health services & Community Services \\
\hline
\end{tabular}

Four different behavior models: semantic, semantic and space, semantic and time, and semantic and space and time were considered in this testing. As it can be seen in Table 9, by considering different behavior models, the number of regular services has significantly reduced for each behavior model. For example, by considering semantic and temporal behavior model, the number of services had decreased from 800 to 309 services. This indicates that considering the user's behavior model can customize the available services for the user by filtering out some irrelevant services.

Table 9. Comparing different behavior types with the number of delivered services

\begin{tabular}{ccc}
\hline Behavior type & $\begin{array}{c}\text { No. of Customized } \\
\text { Services }\end{array}$ & $\begin{array}{c}\text { No. of Regular } \\
\text { Services }\end{array}$ \\
\hline Semantic & 412 & 800 \\
\hline Semantic and space & 215 & 800 \\
\hline Semantic and time & 309 & 800 \\
\hline Semantic and space-time & 54 & 800 \\
\hline
\end{tabular}

\section{CONCLUSIONS}

The proposed methodology was applied to discover interesting knowledge about different behavior types of users as rulesets. The results showed that applying these rulesets could significantly reduce the number of available services and customize the services based on the rules. Therefore, the rule mining process is application dependent and it is required to enrich trajectories with geographic information. Moreover, developing an ontology based model could help to enhance communication between users and the system and service providers could provide the most suitable services to users. Therefore, generating different users' behavior types as new knowledge could be beneficial for different applications in LBS. This knowledge could be exploited to make intelligent predictions about user's future behavior given the time and location of the user. Regarding future work, we are investigating the affect of spatial and temporal uncertainty of discovered association rules and their effect on interpretation. 


\section{REFERENCES}

[1] S. Steiniger, M. Neun, And A. Edwardes, "Foundations Of Location Based Services," Cartouche1Lecture Notes On Lbs, Vol. 1, 2006.

[2] C. S. Jensen, "Research Challenges In Location-Enabled M-Services," In Mobile Data Management, 2002. Proceedings. Third International Conference On, 2002, Pp. 3-7.

[3] R. Ong, M. Wachowicz, M. Nanni, And C. Renso, "From Pattern Discovery To Pattern Interpretation In Movement Data," In Proceedings Of The 2010 Ieee International Conference On Data Mining Workshops, 2010, Pp. 527-534.

[4] V. Bogorny, H. Avancini, B. C. De Paula, C. R. Kuplich, And L. O. Alvares, "Weka-Stpm: A Software Architecture And Prototype For Semantic Trajectory Data Mining And Visualization," Transactions In Gis, Vol. 15, No. 2, Pp. 227-248, 2011.

[5] H. J. Miller, “A Measurement Theory For Time Geography,” Geographical Analysis, Vol. 37, No. 1, Pp. 17-45, 2005.

[6] A. Galton, "Dynamic Collectives And Their Collective Dynamics," Spatial Information Theory, Pp. 300-315, 2005.

[7] M. J. Kraak, “The Space-Time Cube Revisited From A Geovisualization Perspective,” In Proc. 21st International Cartographic Conference, 2003, Pp. 1988-1996.

[8] S. Imfeld, "Time, Point And Space- Towards A Better Analysis Of Wildlife Data In Gis," Phd Thesis, Universitaet Zuerich, 2000.

[9] P. Laube, “Analysing Point Motion, Spatio-Temporal Data Mining Of Geospatial Lifelines," Phd Thesis, Universitz Of Zurich, 2005.

[10] S. Dodge, "Exploring Movement Using Similarity Analysis," Phd Thesis, Universitaet Zuerich, 2011.

[11] J. G. Lee, J. Han, X. Li, And H. Gonzalez, "Traclass: Trajectory Classification Using Hierarchical Region-Based And Trajectory-Based Clustering," Proceedings Of The Vldb Endowment, Vol. 1, No. 1, Pp. 1081-1094, 2008.

[12] H. Cao, N. Mamoulis, And D. W. Cheung, "Discovery Of Periodic Patterns In Spatiotemporal Sequences," Knowledge And Data Engineering, Ieee Transactions On, Vol. 19, No. 4, Pp. 453-467, 2007.

[13] P. Laube, S. Imfeld, And R. Weibel, "Discovering Relative Motion Patterns In Groups Of Moving Point Objects,” International Journal Of Geographical Information Science, Vol. 19, No. 6, Pp. 639668, 2005.

[14] D. Mountain, "Exploring Mobile Trajectories: An Investigation Of Individual Spatial Behaviour And Geographic Filters For Information Retrieval," Phd Thesis, City University, London Department Of Information Science School Of Informatics, 2005.

[15] F. Giannotti, "Mobility, Data Mining And Privacy Understanding Human Movement Patterns From Trajectory Data," 2011, Pp. 4-5.

[16] L. O. Alvares, V. Bogorny, J. A. F. De Macedo, B. Moelans, And S. Spaccapietra, "Dynamic Modeling Of Trajectory Patterns Using Data Mining And Reverse Engineering," In Tutorials, Posters, Panels And Industrial Contributions At The 26th International Conference On Conceptual ModelingVolume 83, 2007, Pp. 149-154.

[17] S. Spaccapietra, C. Parent, M. L. Damiani, J. A. De Macedo, F. Porto, And C. Vangenot, "A Conceptual View On Trajectories," Data \& Knowledge Engineering, Vol. 65, No. 1, Pp. 126-146, Apr. 2008.

[18] L. O. Alvares, V. Bogorny, B. Kuijpers, J. A. F. De Macedo, B. Moelans, And A. Vaisman, "A Model For Enriching Trajectories With Semantic Geographical Information," In Proceedings Of The 15th Annual Acm International Symposium On Advances In Geographic Information Systems, 2007, P. 22.

[19] C. Parent, S. Spaccapietra, And E. Zimányi, Conceptual Modeling For Traditional And SpatioTemporal Applications: The Mads Approach. Springer Science \& Business Media, 2006.

[20] Z. Yan, J. Macedo, C. Parent, And S. Spaccapietra, "Trajectory Ontologies And Queries," Transactions In Gis, Vol. 12, No. S1, Pp. 75-91, 2008.

[21] A. Mousavi And A. J. S. Hunter, "Using Ontology Based Knowledge Discovery In Location Based Services," Presented At The The 2012 Autocarto International Symposium On Automated Cartography, Columbus, Ohio, 2012.

[22] R. H. Güting And M. Schneider, Moving Objects Databases. Morgan Kaufmann Pub, 2005. 
[23] N. Pelekis, Y. Theodoridis, S. Vosinakis, And T. Panayiotopoulos, "Hermes-A Framework For Location-Based Data Management," Advances In Database Technology-Edbt 2006, Pp. 1130-1134, 2006.

[24] I. I. Tsoukatos And D. Gunopulos, "Efficient Mining Of Spatiotemporal Patterns," Advances In Spatial And Temporal Databases, Pp. 425-442, 2001.

[25] Y. Li, J. Han, And J. Yang, "Clustering Moving Objects," In Proceedings Of The Tenth Acm Sigkdd International Conference On Knowledge Discovery And Data Mining, 2004, Pp. 617-622.

[26] M. Nanni And D. Pedreschi, “Time-Focused Clustering Of Trajectories Of Moving Objects,” Journal Of Intelligent Information Systems, Vol. 27, No. 3, Pp. 267-289, 2006.

[27] S. Spaccapietra, C. Parent, M. L. Damiani, J. A. De Macedo, F. Porto, And C. Vangenot, "A Conceptual View On Trajectories," Data \& Knowledge Engineering, Vol. 65, No. 1, Pp. 126-146, 2008.

[28] M. Baglioni, J. A. Fernandes De Macêdo, C. Renso, R. Trasarti, And M. Wachowicz, "Towards Semantic Interpretation Of Movement Behavior," In Advances In Giscience, 2009, Pp. 271-288.

[29] R. Trasarti, S. Rinzivillo, F. Pinelli, M. Nanni, A. Monreale, C. Renso, D. Pedreschi, And F. Giannotti, "Exploring Real Mobility Data With M-Atlas," Machine Learning And Knowledge Discovery In Databases, Pp. 624-627, 2010.

[30] B. Smith And D. M. Mark, "Ontology And Geographic Kinds," In 8th International Symposium On Spatial Data Handling (Sdh'98), Vancouver, Canada, 1998, Pp. 308-320.

[31] M. Charest And S. Delisle, "Ontology-Guided Intelligent Data Mining Assistance: Combining Declarative And Procedural Knowledge,” In Artificial Intelligence And Soft Computing, 2006, Pp. 914.

[32] J. Phillips And B. G. Buchanan, "Ontology-Guided Knowledge Discovery In Databases," In Proceedings Of The 1st International Conference On Knowledge Capture, 2001, Pp. 123-130.

[33] A. Bernstein, S. Hill, And F. Provost, "Intelligent Assistance For The Data Mining Process: An Ontology-Based Approach,” Nyu Working Paper No. 2451/14146, 2002.

[34] M. Bauer And S. Baldes, "An Ontology-Based Interface For Machine Learning," In Proceedings Of The 10th International Conference On Intelligent User Interfaces, 2005, Pp. 314-316.

[35] M. Cannataro And C. Comito, “A Data Mining Ontology For Grid Programming," Proceedings Of (Sempgrid2003), Pp. 113-134, 2003.

[36] A. Mousavi And A. J. S. Hunter, "Pointers Extraction Of Trajectory Data For Semantic Knowledge Discovery," Presented At The Giscience 2012 - Seventh International Conference On Geographic Information Science, Columbus, Ohio, 2012.

[37] R. Agrawal, T. Imieliński, And A. Swami, "Mining Association Rules Between Sets Of Items In Large Databases,” In Acm Sigmod Record, 1993, Vol. 22, Pp. 207-216.

[38] R. Agrawal And R. Srikant, "Fast Algorithms For Mining Association Rules," In Proc 20th Int Conf Very Large Data Bases Vldb, 1994, Vol. 1215, Pp. 487-499. 